\title{
A Method for Queue Length Estimation in an Urban Street Network Based on Roll Time Occupancy Data
}

\author{
Dongfang $\mathrm{Ma}^{1}{ }^{1}$ Dianhai Wang, ${ }^{1}$ Yiming Bie, ${ }^{2}$ \\ Feng Sun, ${ }^{3}$ and Sheng Jin ${ }^{1}$ \\ ${ }^{1}$ College of Civil Engineering and Architecture, Zhejiang University, Hangzhou 310058, China \\ ${ }^{2}$ School of Transportation Science and Engineering, Harbin Institute of Technology, Harbin 150091, China \\ ${ }^{3}$ College of Traffic and Transportation, Jilin University, Changchun 130022, China
}

Correspondence should be addressed to Dianhai Wang, wangdianhai@zju.edu.cn

Received 15 May 2012; Revised 19 August 2012; Accepted 26 August 2012

Academic Editor: Wuhong Wang

Copyright (C) 2012 Dongfang Ma et al. This is an open access article distributed under the Creative Commons Attribution License, which permits unrestricted use, distribution, and reproduction in any medium, provided the original work is properly cited.

\begin{abstract}
A method estimating the queue length in city street networks was proposed using the data of roll time occupancy. The key idea of this paper is that when the queue length in front of the queue detector becomes longer, the speeds of the following vehicles to pass through the detector will become smaller, resulting in higher occupancy with constant traffic intensity. Considering the relationship between queue lengths and roll time occupancy affected by many factors, such as link length, lane width, lane number, and bus ratio, twelve different conditions were designed, and the traffic data under different conditions was obtained using VISSIM simulation. Based on the analysis of simulation data, an S-type logistic model was decided to develop for the relationship between queue lengths and roll time occupancy, and the fitting equations were obtained under the twelve simulation situations. The average model for the relationship between queue lengths and roll time occupancy was presented by successive multiple linear regression with the fitting equation parameters and simulation parameters, and the estimation model for queue length was presented through analyzing the equation of the average relation model.
\end{abstract}

\section{Introduction}

With traffic congestion worsening in urban areas, a growing number of signalized intersections are being operated in oversaturated conditions, and the queue lengths at some roads are even approaching the link lengths during peak hours, leading to spillovers [1,2]. As the influence of spillovers in city street networks is significant [3], monitoring the traffic state of the roads and estimating the conditions under which a spillover will occur are important.

It has long been recognized that the queue length can represent the road traffic state intuitively, and it is the most common index to identify spillovers. Over the years, many 


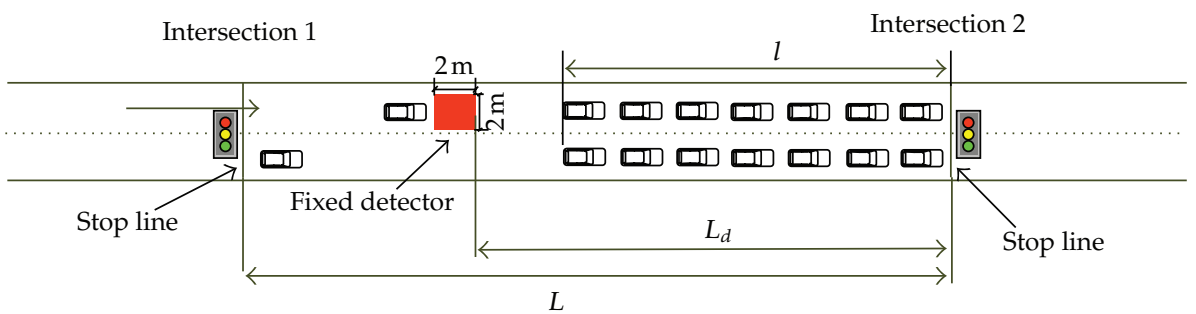

Figure 1: Location of queue detector.

researchers have dedicated themselves to this topic and three types of queue estimation models have been developed. The first one, which is based on the behavior of traffic shockwaves, was first proposed by Lighthill and Whitham for uninterrupted flow [4] and later improved and expanded by a number of researchers to signalized intersections [5-8]. The second one demonstrated by Webster [9] is based on the analysis of cumulative traffic input-output to a signal link. Later, it was improved by some researchers including Daganzo [10], Akcelik [11], and Vigos et al. [12]. As high-resolution traffic signal data, such as secondby-second detector data, vehicle-detector actuation events, and signal phase change events, is becoming increasingly available in recent years; a new model for estimating the maximum queue length during every cycle was developed by Liu et al. [13, 14].

Known probability distribution of traffic flow arrival rule is the premise for the first kind methods to estimate the queue length, and they seemly become no more applicable if the change of traffic state is very complicated or the traffic flow arrival rule does not suit to any known probability distribution. The second class methods can be used to estimate queue length both for macroscopic and microscopic levels; however, constructing the cumulative curve and solving the queue length in these methods are very arduous. In the third type models, the three break points which indicate a change in traffic state are difficult to identify if the residual queue length at the beginning of the red phase is greater than the distance between the stop line and the detector location. Moreover, traffic-wave theory, with its premise of continuous flow, is not suitable for practical application, as the discontinuity of traffic flow in urban street networks is significant under the influence of signal controls [15]; thus, methods taking traffic-wave theory as their basis are impracticable.

To close these gaps mentioned above, this paper analyzes the relationship between queue length and roll time occupancy, using the data collected by VISSIM simulations, and then develops a new method to estimate the queue length using the data of roll time occupancy. The finding of this paper may provide oversaturated arterials a basis to identify spillover conditions.

\section{Computation of Roll Time Occupancy}

With the aim of obtaining traffic data for predicting the road traffic state, fixed loop detectors are placed sufficiently upstream from the intersection stop line in SCOOT (Split, Cycle, and Offset Optimization Technique) and other systems [16]. These detectors can be called as queue detectors or advanced detectors, whose locations can be illustrated as in Figure 1 .

The most common type of queue detector is a loop detector, which can provide traffic flow information in the form of pulse data, and is shown in Figure 2. The upper bars represent 


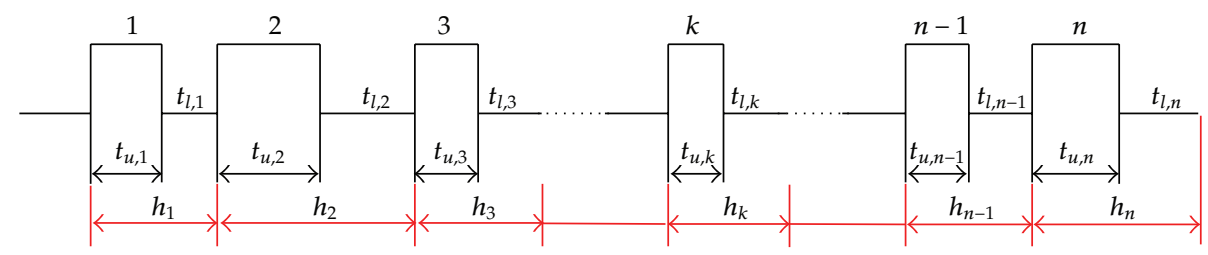

Figure 2: Pulse information from a loop detector.

the state where the detector is occupied by one vehicle, and the duration corresponds to occupancy time of this vehicle; the lower bars stand for the gaps between the rear of the forward vehicle and the start of the following vehicle. The sum of the durations of an upper and the following lower bars is the headway between two successive vehicles.

$t_{u, k}$, the occupancy time of the $k$ th vehicle, can be obtained from the vehicle length and average speed data using:

$$
t_{u, k}=\frac{\left(L_{k}+L_{q}\right)}{u_{k}}
$$

where $u_{k}$ is the average speed of the $k$ th vehicle when passing through the queue detector $(\mathrm{m} / \mathrm{s}) ; L_{k}$ is the length of vehicle $k(\mathrm{~m}) ; L_{q}$ is the length of queue detector $(\mathrm{m})$, which is about $2.0 \mathrm{~m}$ in actual application.

As shown in (2.1), the lower the value of $u_{k}$, the higher the value of $t_{u, k}$; meanwhile, the expected speed of all drivers is the free flow speed, and a lower speed indicates a higher queue length (i.e., the state on the road is congested). As a consequence, it can be deduced that the occupancy time of one vehicle may characterize the traffic condition around the queue detector at the time when the vehicle is passing through and the queue length can be estimated by analyzing the data of occupancy.

Occupancy is the ratio of the sum of the durations of the upper bars during a given period (denoted as $T$ ) to the entire duration of that period. Aiming to identify the traffic condition and estimate the queue length at some roads instantaneously, the idea of roll time occupancy is proposed, which could indicate the traffic condition around a queue detector continuously within an interval of $\Delta t$. Figure 3 illustrates the statistical method of calculating occupancy time, taking $T=5 \Delta t$ as an example.

The roll time occupancy in period $i, o_{i}$, is given by

$$
o_{i}=\frac{t_{i}}{T}
$$

Here, $\Delta t$ is the time of rolling step (s), usually set to $1 \mathrm{~s}$ in order to obtain the traffic state around the queue detector each second; $T$ is the time length of each period for counting roll time occupancy (s), which is usually set to $5 \mathrm{~s}$ in real application; $t_{i}$ is the time for which queue detectors are occupied by vehicles in period $i$ (s).

As mentioned above, the traffic condition will become congested around the queue detectors as the increasing of occupancy, and there is a positive correlation between roll time occupancy and queue length for known traffic intensity. Thus, the queue length on some roads can be estimated by analyzing the data of roll time occupancy. 


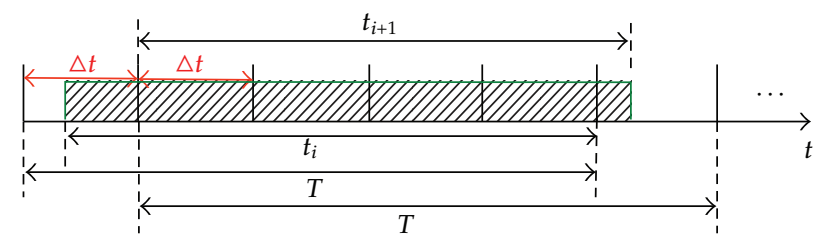

Figure 3: Calculation of roll time occupancy.

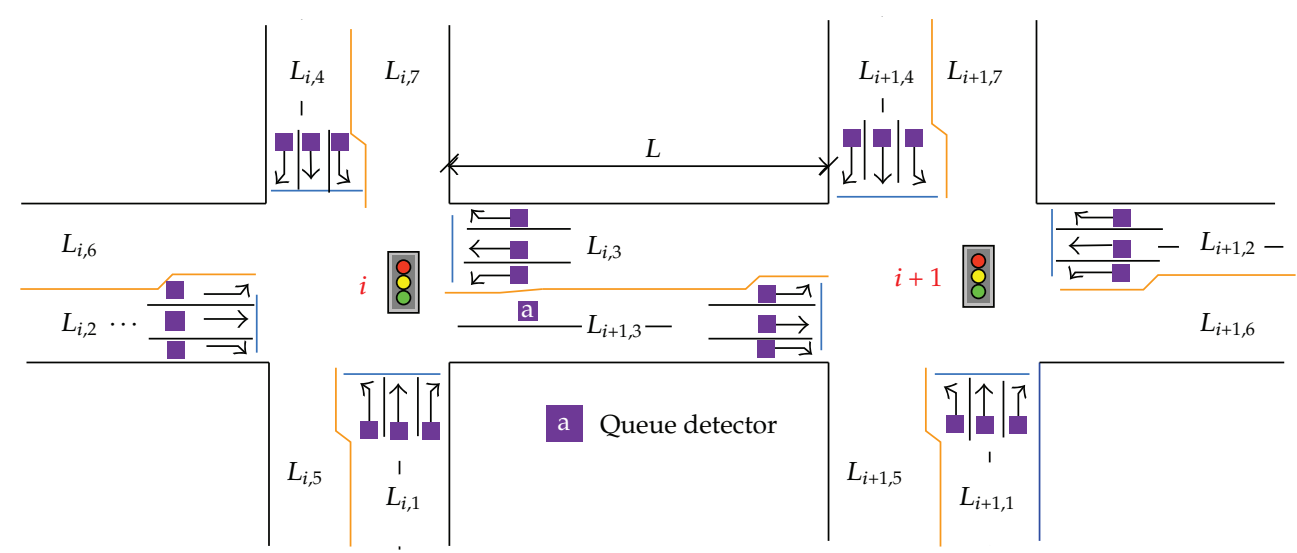

Figure 4: Experimental road grid for the simulation.

\section{Relationship between Queue Length and Roll Time Occupancy}

Generally, higher roll time occupancy corresponds to a longer queue length, and the relationship between the two can be obtained from traffic data.

\subsection{Date Collection and Relevant Parameters}

Traffic data collected by a queue detector over a given period of time can vary wildly under the influence of different traffic flow and road conditions. In this paper, traffic data was collected using a VISSIM simulation under different conditions. The experimental road grid is shown in Figure 4.

Here, $i+1$ is the downstream intersection, and $L_{i+1,3}$ is the link of interest, where a queue detector is placed at the inside lane. The distance between the queue detector and the upstream intersection is $50 \mathrm{~m}$, and the link lengths of the six approaches, other than $L_{i+1,3}$ and $L_{i, 3}$, whose parameters vary and are given later, are listed in Table 1.

The signal timings of the two intersections are also presented in the Table 1, with phases 1, 2, 3, and 4 representing the East-West through phase, the East-West left turn phase, the South-North through phase, and the South-North left turn phase, respectively. The key parameters in VISSIM are set as follows: (1) the expected speeds of cars and buses are $15 \mathrm{~m} / \mathrm{s}$ and $10 \mathrm{~m} / \mathrm{s}$, respectively; (2) the saturated flow is $1,800 \mathrm{veh} / \mathrm{h}$ for all lanes; (3) the traffic volume is set to be $1,200 \mathrm{veh} / \mathrm{h}$ for all approaches; (4) the length of channelization segment is $71 \mathrm{~m}$ for all approaches; (5) the ratio of right-turning to through to left-turning vehicles on all approaches is $1: 2: 1 ;(6)$ the random seed is $41 ;(7)$ when the speed is less than $0.28 \mathrm{~m} / \mathrm{s}$, 
Table 1: Link lengths and phase sequences of the two intersections.

\begin{tabular}{|c|c|c|c|c|c|c|c|}
\hline \multirow{2}{*}{ Intersection } & \multicolumn{3}{|c|}{ Link length $(\mathrm{m})$} & \multicolumn{4}{|c|}{ Green time (s) } \\
\hline & $\begin{array}{c}\text { Approach } \\
\text { no. } 1\end{array}$ & $\begin{array}{c}\text { Approach } \\
\text { no. } 2\end{array}$ & $\begin{array}{c}\text { Approach } \\
\text { no. } 4\end{array}$ & $\begin{array}{c}\text { Phase no. } \\
1\end{array}$ & $\begin{array}{c}\text { Phase no. } \\
2\end{array}$ & $\begin{array}{c}\text { Phase no. } \\
3\end{array}$ & $\begin{array}{c}\text { Phase no. } \\
4\end{array}$ \\
\hline$i$ & 550 & 550 & 550 & 32 & 20 & 36 & 20 \\
\hline$i+1$ & 550 & 580 & 550 & 24 & 21 & 32 & 31 \\
\hline
\end{tabular}

Table 2: Parameters relating to link no. 3 in the simulation environment.

\begin{tabular}{lccccccccc}
\hline Condition number & $L(\mathrm{~m})$ & $n$ & $w_{d}(\mathrm{~m})$ & $r(\%)$ & Condition number & $L(\mathrm{~m})$ & $n$ & $w_{d}(\mathrm{~m})$ & $r(\%)$ \\
\hline 1 & 300 & 2 & 3.5 & 5 & 7 & 300 & 2 & 3.5 & 5 \\
2 & 350 & 2 & 3.5 & 5 & 8 & 300 & 2 & 3.5 & 10 \\
3 & 400 & 2 & 3.5 & 5 & 9 & 300 & 2 & 3.5 & 15 \\
4 & 350 & 1 & 3.5 & 5 & 10 & 300 & 2 & 3 & 5 \\
5 & 300 & 2 & 3.5 & 5 & 11 & 300 & 2 & 3.5 & 5 \\
6 & 300 & 3 & 3.5 & 5 & 12 & 300 & 2 & 3.75 & 5 \\
\hline
\end{tabular}

we define the queue as building-up, and when the speed is greater than $1.39 \mathrm{~m} / \mathrm{s}$, we define the queue as dissipating; (8) the simulation time is 3,600 s.

With known traffic demand, the operational characteristics of the traffic flow on the stretch of road $L_{i+1,3}$ will differ depending on the parameters in VISSIM, including link length $(L)$, lane width $(w)$, number of lanes $(n)$, and bus ratio $(r)$. A total of twelve simulation conditions were designed for this paper; the parameters of which are listed in Table 2.

The following section will collect the traffic data under different conditions and analyze the relationship between the queue lengths on the interest link and roll time occupancy collected by queue detectors; Sections 3.3 and 3.4 will discuss the relationship between the parameters of the new model and these four simulation parameters mentioned above.

\subsection{Queue Length and Roll Time Occupancy}

The signal timing plans of the two intersections were set using the VAP module of VISSIM, and the traffic data was obtained regarding the roll time occupancy and maximum queue length with a rolling step of $\Delta t$ by adjusting the data output interval. Figure 5 illustrates the relationship between roll time occupancy and queue length under simulation condition no. 1.

Similar relationship models between queue length and roll time occupancy can be obtained by changing the simulation conditions, and then an S-type logistic model for the relationship was developed based on the analysis of the points in Figure 5. The conceptual shape of the logistic model is shown in Figure 6 and is devised as follows:

$$
o=o_{\min }+\frac{o_{\max }-o_{\min }}{1+e^{\left[-b\left(l-l_{w}\right)\right]}}
$$

where $o_{\min }$ is the minimum roll time occupancy; $o_{\max }$ is the maximum roll time occupancy; $l$ is the queue length of a road where spillovers appear regularly $(\mathrm{m}) ; l_{w}$ is the queue length at the deflection point of the curve and $b$ determines the slope of the curve. 


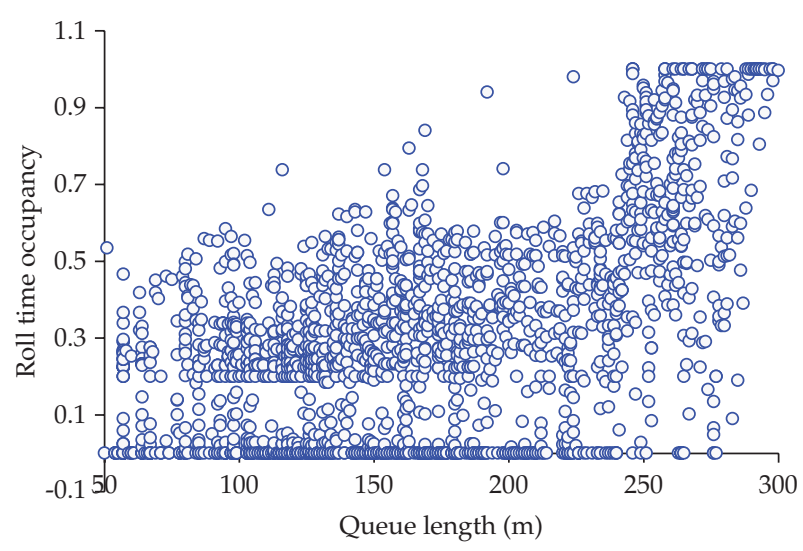

Figure 5: Relationship between $l$ and $o$ with $w_{d}=3.5 \mathrm{~m}$.

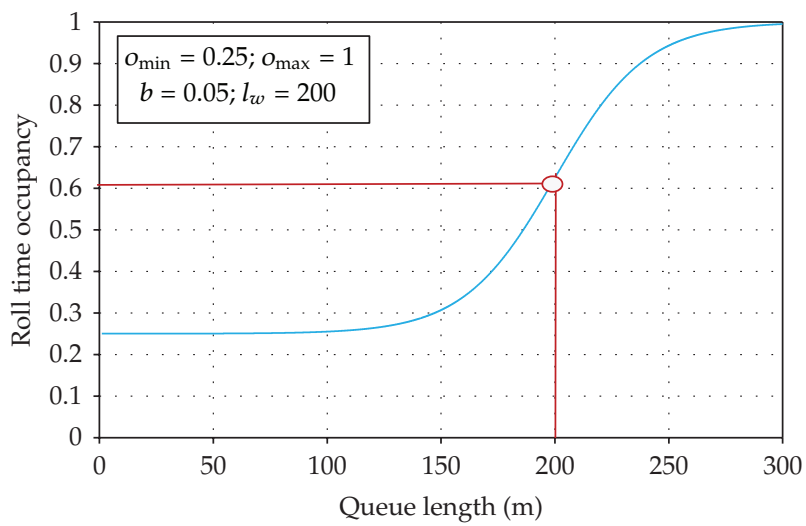

Figure 6: Conceptual shape of $l$ versus $o$.

It is necessary to define the upper and lower values of the asymptotes, that is, the values of $o_{\max }$ and $o_{\min } . O_{\min }$ is the smallest roll time occupancy under a given simulation environment. The parameter, $o_{\max }$, which is calculated based on the simulation conditions, is the maximum roll time occupancy. It is equal to 1 , which can be confirmed from the definition of roll time occupancy. Given the initial parameters and the assumed form of the model, it is possible to calibrate the model of each simulation environment by applying the best-fitting parameters, based on the data collected under each of the twelve simulation conditions. The result of this calibration for the simulation situation in Figure 5 is shown in Figure 7.

The S-shape of the curve is also superimposed over the entire range of queue length, up to $300 \mathrm{~m}$. The data points for the simulation environment are presented in order to show the "goodness-of-fit" of the model to the data. In this study, the fitting relationship between $o$ and $l$ is

$$
o=0.2858+\frac{0.7142}{1+e^{[-0.0552(l-251.3985)]}}, \quad R=0.7529 .
$$

Using the same method, equations were fitted for the twelve simulation situations, as given in Table 3. 


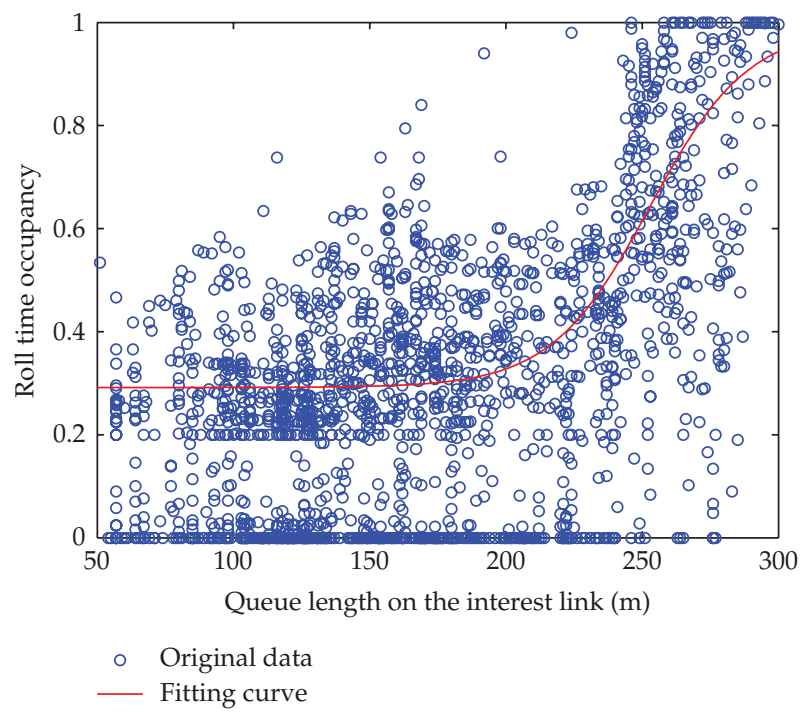

Figure 7: Calibration of $S$-curve between $l$ and $o$.

Table 3: Fitting equations and correlation coefficients under different simulation conditions.

\begin{tabular}{lcccccc}
\hline Number & Fitted equations & $R$ & Number & Fitted equations & $R$ \\
\hline 1 & $o=0.2858+\frac{0.7142}{1+e^{[-0.0552(l-251.3985)]}}$ & 0.7529 & 7 & $o=0.2858+\frac{0.7142}{1+e^{[-0.0552(l-251.3985)]}}$ & 0.7529 \\
2 & $o=0.2801+\frac{0.7199}{1+e^{[-0.0516(l-299.76)]}}$ & 0.8716 & 8 & $o=0.3082+\frac{0.6918}{1+e^{[-0.0953(l-247.7518)]}}$ & 0.8000 \\
3 & $o=0.2499+\frac{0.7501}{1+e^{[-0.0375(l-327.7079)]}}$ & 0.8319 & 9 & $o=0.3349+\frac{0.6651}{1+e^{[-0.0949(l-252.7541)]}}$ & 0.7671 \\
4 & $o=0.3223+\frac{0.6777}{1+e^{[-0.0572(l-278.2494)]}}$ & 0.7652 & 10 & $o=0.2917+\frac{0.6998}{1+e^{[-0.0552(l-253.8729)]}}$ & 0.7409 \\
5 & $o=0.303+\frac{0.696}{1+e^{[-0.086(l-249.0782)]}}$ & 0.7758 & 11 & $o=0.3330+\frac{0.667}{1+e^{[-0.0979(l-255.6569)]}}$ & 0.7416 \\
6 & $o=0.2917+\frac{0.7083}{1+e^{[-0.0797(l-251.7239)]}}$ & 0.7847 & 12 & $o=0.2805+\frac{0.7195}{1+e^{[-0.0749(l-247.9341)]}}$ & 0.7953 \\
\hline
\end{tabular}

Analyzing the fitting equations under different simulation conditions, the values of $o_{\min }$ are similar in each case; however, the values of $b$ and $l_{w}$ vary greatly. Consequently, the four parameters, $L, w_{d}, n$, and $r$ can be said to strongly influence the values of $b$ and $l_{w}$, and it is thus necessary to obtain the relationship between $o$ and $l$ under normal conditions.

\subsection{Regression Analysis for Parameter $b$}

Parameter $b$ represents the geometric complexity for constant traffic intensity. The complexity is calculated from the link length, lane width, number of lanes, and bus ratio. A macroscopic model was developed for parameter $b$ through a multiple-stepwise linear regression of the four parameters under each simulation situation. Two models were produced by the statistical software, SPSS [17]; a summary of which is presented in Table 4. 
Table 4: Model summaries for parameter $b$.

\begin{tabular}{lcccc}
\hline Model & $R$ & $R^{2}$ & Adjusted $R^{2}$ & Std. error \\
\hline 1 & $0.663^{\mathrm{a}}$ & 0.440 & 0.384 & 0.0258196 \\
2 & $0.813^{\mathrm{b}}$ & 0.661 & 0.586 & 0.0121482 \\
\hline
\end{tabular}

aredictors: (constant), bus ratio; ${ }^{b}$ Predictors: (constant), bus ratio, link length.

Table 5: ANOVA for parameter $b$.

\begin{tabular}{ccccccc}
\hline & Model & Sum of squares & $\mathrm{df}$ & Mean square & $F$ & Sig. \\
\hline \multirow{2}{*}{1} & Regression & 0.002 & 1 & 0.002 & \multirow{2}{*}{7.855} & $0.019^{\mathrm{a}}$ \\
\hline & Residual & 0.002 & 10 & 0.000 & & \\
\hline \multirow{2}{*}{2} & Total & 0.004 & 11 & & \multirow{2}{*}{0.785} & $0.008^{\mathrm{b}}$ \\
\hline & Regression & 0.003 & 2 & 0.001 & & \\
\hline
\end{tabular}

${ }^{\mathrm{a}}$ Predictors: (constant), bus ratio; ${ }^{\mathrm{b}}$ Predictors: (constant), bus ratio, link length.

Table 6: Coefficients for parameter $b$.

\begin{tabular}{|c|c|c|c|c|c|c|}
\hline & \multirow{2}{*}{ Model } & \multicolumn{2}{|c|}{ Unstandardized coefficients } & \multirow{2}{*}{$\begin{array}{c}\text { Standardized coefficients } \\
\beta\end{array}$} & \multirow{2}{*}{$t$} & \multirow{2}{*}{ Sig. } \\
\hline & & $B$ & Std. Error & & & \\
\hline \multirow{2}{*}{1} & Constant & 0.041 & 0.010 & & 4.151 & .002 \\
\hline & Bus Ratio & 0.403 & 0.144 & 0.663 & 2.803 & .019 \\
\hline \multirow{3}{*}{2} & Constant & 0.134 & 0.039 & & 3.428 & .008 \\
\hline & Bus Ratio & 0.337 & 0.121 & 0.555 & 2.787 & .021 \\
\hline & Link length & 0.00028 & 0.000 & -0.483 & -2.425 & .038 \\
\hline
\end{tabular}

As the determination coefficient $\left(R^{2}\right)$ of model no. 1 is less than 0.5 , the goodness-of-fit is not acceptable. However, the determination coefficient of model no. 2 is 0.586 , indicating an acceptable goodness-of-fit [18]. The variance analysis is presented in Table 5.

From the variance analysis of model no. 2 in Table 5, it can be seen that the model has very high precision $(F=8.785, P=0.008<0.01)$. Moreover, all of the values of $P$ for the three terms in $t$-test are less than 0.05 (see Table 6), which indicates that all three regression coefficients are significant with $\alpha=0.05$. The regression coefficients are tabulated in Table 6 .

In model no. 2, the regression coefficients of $r, L$, and the constant term are 0.337, 0.00028 , and 0.134 , respectively. Therefore, the regression equation can be expressed as, which indicates that the value of $b$ is only affected by $L$ and $r$ the following:

$$
b=-0.000228 L+0.337 r+0.134 \text {. }
$$

\subsection{Regression Analysis for Parameter $l_{w}$}

It is also necessary to replace the value of $l_{w}$ (the queue length at the inflection point of the $S$-curve in Figure 6) with a regression model with the four aforementioned parameters under the twelve simulation situations. One model is produced by SPSS, as summarized in Table 7.

The determination coefficient $\left(R^{2}\right)$ of this model is 0.959 ; the goodness-of-fit is acceptable. The variance analysis is listed in Table 8. 
Table 7: Model summary for parameter $l_{w}$.

\begin{tabular}{lcccc}
\hline Model & $R$ & $R^{2}$ & Adjusted $R^{2}$ & Std. error \\
\hline 1 & $0.979^{\mathrm{a}}$ & 0.959 & 0.955 & 5.3884779 \\
\hline a Predictors: (constant), link length. & & &
\end{tabular}

Table 8: ANOVA for parameter $l_{w}$.

\begin{tabular}{lcccccc}
\hline \multicolumn{2}{c}{ Model } & Sum of squares & df & Mean square & $F$ & Sig. \\
\hline 1 & Regression & 6737.63 & 1 & 6737.63 & 232.05 & $.000^{\text {a }}$ \\
\hline & Residual & 290.36 & 10 & 29.04 & & \\
\hline \multicolumn{2}{c}{ Total } & 7027.99 & 11 & &
\end{tabular}

Table 9: Coefficients for parameter $l_{w}$.

\begin{tabular}{|c|c|c|c|c|c|c|}
\hline \multirow{2}{*}{\multicolumn{2}{|c|}{ Model }} & \multicolumn{2}{|c|}{ Unstandardized coefficients } & \multirow{2}{*}{$\begin{array}{c}\text { Standardized coefficients } \\
\beta\end{array}$} & \multirow{2}{*}{$t$} & \multirow{2}{*}{ Sig. } \\
\hline & & $B$ & Std. error & & & \\
\hline \multirow{2}{*}{1} & (Constant) & 23.378 & 15.874 & & 1.473 & .172 \\
\hline & Link length & 0.760 & 0.050 & 0.979 & 15.233 & .000 \\
\hline
\end{tabular}

The values of $F$ and $P$ in this model are 232.047 and 0.000 , respectively, which indicates that the precision of this regression model is very high. Moreover, the constant term does not affect $l_{w}$ significantly $(P=0.172>0.05)$ with $\alpha=0.05$ (as shown in Table 9), so it could be deduced that the constant term should be rejected from the regression model. The coefficients of parameter $l_{w}$ are listed in Table 9.

The coefficients of the constant term and link length are 23.378 and 0.0760 , respectively, and the regression equation is given as follows:

$$
l_{w}=0.706 L, \quad R^{2}=0.9793 .
$$

Equation (3.4) suggests that $l_{w}$ is only affected by $L$.

When there is no real-time data on queue length, it is possible to utilize an average model to determine the relationship between roll time occupancy and queue length, which may represent the average conditions of a variety of links and provide a method to estimate the queue length in general conditions. In this study, the mean of $o_{\min }$ was used in the twelve cases as the general value, and substitute (3.3) and (3.4) into (3.1), then, the average model for the roll time occupancy can be obtained:

$$
o=0.3056+\frac{1-0.3056}{1+e^{[-(0.000228 L+0.337 r+0.134)(l-0.760 L)]}} .
$$

When the link length is $300 \mathrm{~m}$, the number of lanes is 1 , and the bus ratio is $5 \%$, the average inflection roll time occupancy (i.e., the corresponding roll time occupancy when $l$ is equal to $l_{w}$ ) is found to be 0.6662 . 


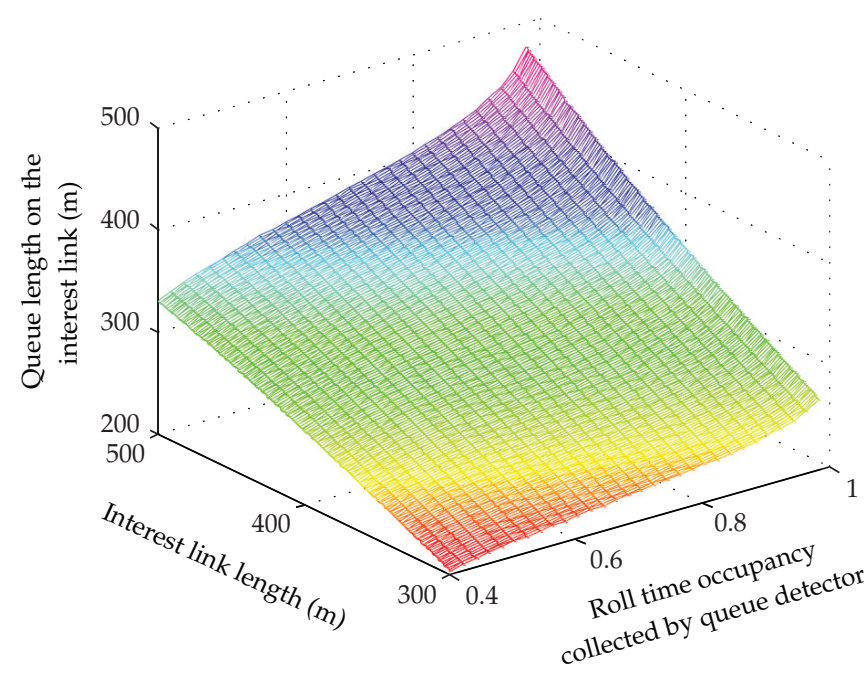

Figure 8: Influence of link length.

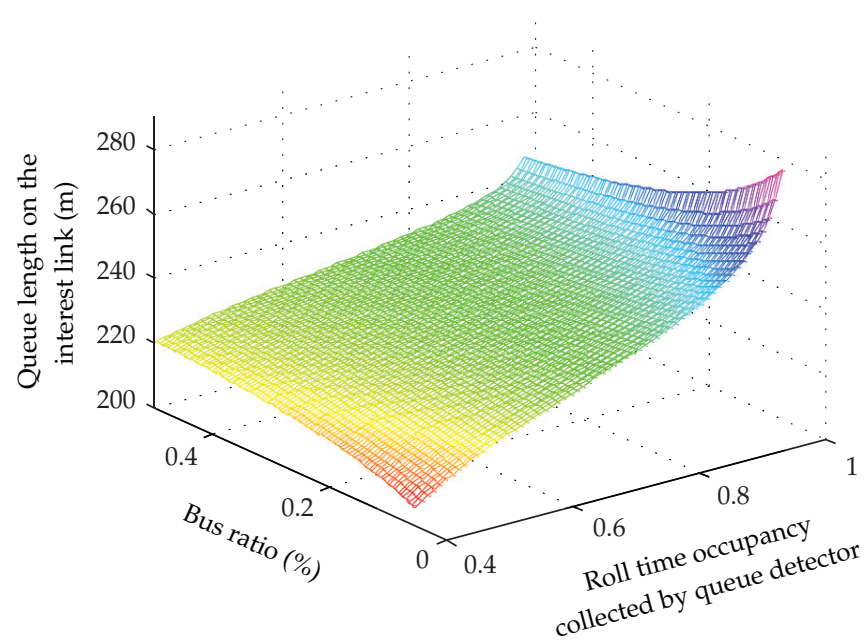

Figure 9: Influence of bus ratio.

\section{Queue Length Model with Roll Time Occupancy}

An estimation model for queue length using the data of roll time occupancy can be obtained by transforming (3.5), which gives

$$
l_{i}=0.706 L+\frac{1}{0.000228 L-0.337 r-0.134} \ln \left(\frac{1-o_{i}}{o_{i}-0.3056}\right) .
$$

With known traffic conditions of one road, the influences of link length and bus ratio on the relationship between queue length and roll time occupancy are shown in Figures 8 and 9. (The bus ratio in Figure 8 is 5\%; the link length in Figure 9 is $350 \mathrm{~m}$ ). 
The analysis of Figures 8 and 9 shows that

(1) the relation forms between queue length and roll time occupancy keep invariant under the influence of link length and bus ratio;

(2) the queue length is directly proportional to the link length when other parameters are constant, and the influence degree will become higher with roll time occupancy becoming greater;

(3) with lower roll time occupancy, the influence of bus ratio on the relationship is insignificant. The reason may be that lower roll time occupancy represents lower traffic flow, and the difference of occupancy time between buses and cars when they passing through the queue detector is not obvious, which indicates that the occupancy time in every interval is almost uniform;

(4) high roll time occupancy may be caused by two conditions: (a) the queue length approaches to the link length, and all vehicles pass through the detector with congested speed; (b) if the farthest point of queues is close to the queue detector, the speeds of vehicles passing through the detector should approach to the congested speed, and the average occupancy time per vehicle will become larger under the influence of bus ratio; based on the latter condition, it is concluded that the queue length will decrease with the increasing of bus ratio with an identically higher roll time occupancy (seen as in Figure 9).

Furthermore, in order to validate the queue length estimation model proposed in this paper, another simulation was finished with the same experimental road grid and signal timing scheme except that the bus ratio and interest length link are $8 \%$ and $375 \mathrm{~m}$, respectively. The estimated value of queue length can be calculated based on (4.1) and the roll time occupancy collected by VISSIM. The average error of the 3,600 data series is about $23.14 \%$, which indicates the precision of this new model is accepted.

\section{Conclusions}

In this paper, a method of estimating queue length based on fixed detector outputs was proposed, by focusing on the relationship between the roll time occupancy at a particular position and the queue length on one road. Twelve simulation conditions were designed using different combinations of these four parameters, including link length, lane width, number of lanes, and bus ratio. Based on the analysis of the simulation data, an S-type logistic model was chosen to represent the relationship between queue lengths and roll time occupancy, and equations were fitted for the twelve simulation situations. Then, the average model for roll time occupancy was achieved using successive multiple-stepwise linear regressions with the parameters of the fitting equations and simulation environments. Finally, a new estimation model of queue length based on roll time occupancy was obtained by transforming the average model, which can provide oversaturated arterials a basis to identify spillover conditions.

Considering the effect of traffic signal control, vehicles departed from the upstream intersection will pass through the detector with strong discontinuity and the queue detector would be idled for a long interval, which may bring the result that some lower roll time occupancy corresponds to higher queue length. Furthermore, there may be lane changing near the queue detector. The detector may not be occupied even if the queue length is larger 
than the distance between the stop line and the queue detector, which also can lead to lower roll time occupancy corresponding to higher queue length. The estimation accuracy of the method for estimating queue length proposed in this paper can be improved, if the invalid data caused by the two factors mentioned above can be eliminated accurately.

\section{Acknowledgment}

This research was supported in part by the National High Technology Research and Development Program of China (no. 2011AA110304).

\section{References}

[1] N. Geroliminis and A. Skabardonis, "Identification and analysis of queue spillovers in city street networks," IEEE Transactions on Intelligent Transportation Systems, vol. 12, no. 4, pp. 1107-1115, 2011.

[2] W. W. Guo, W. H. Wang, G. Wets et al., "Influence of stretching-segment storage length on urban traffic flow in signalized intersection," International Journal of Computational Intelligence Systems, vol. 4, no. 6, pp. 1401-1406, 2011.

[3] D. C. Gazis, "Optimal control of a system of oversaturated intersections," Operations Research, vol. 12, no. 6, pp. 815-831, 1964.

[4] M. J. Lighthill and G. B. Whitham, “On kinematic waves. I: flood movement in long rivers II: a theory of traffic flow on long crowded roads," Proceedings of the Royal Society. London A, vol. 229, pp. 281-316, 1955.

[5] G. Stephanopoulos and P. G. Michalopoulos, "Modelling and analysis of traffic queue dynamics at signalized intersections," Transportation Research A, vol. 13, no. 5, pp. 295-307, 1979.

[6] P. G. Michalopoulos and G. Stephanopoulos, "Oversaturated signal systems with queue length constraints-II. Systems of intersections," Transportation Research, vol. 11, no. 6, pp. 423-428, 1977.

[7] R. Akcelik and N. M. Rouphail, "Overflow queues and delays with random and platooned arrivals at signalized intersections," Journal of Advanced Transportation, vol. 28, no. 3, pp. 227-251, 1994.

[8] A. Sharma, D. M. Bullock, and J. A. Bonneson, "Input-output and hybrid techniques for real-time prediction of delay and maximum queue length at signalized intersections," Transportation Research Record, no. 2035, pp. 69-80, 2007.

[9] F. V. Webster, Traffic Signal Settings, Road Research Technical Paper No. 39, Research Laboratory, London, UK, 1958.

[10] C. F. Daganzo, "Queue spillovers in transportation networks with a route choice," Transportation Science, vol. 32, no. 1, pp. 3-11, 1998.

[11] R. Akcelik, A Queue Model for HCM 2000, ARRB, Melbourne, Australia, 1999.

[12] G. Vigos, M. Papageorgiou, and Y. Wang, "Real-time estimation of vehicle-count within signalized links," Transportation Research C, vol. 16, no. 1, pp. 18-35, 2008.

[13] H. X. Liu, X. Wu, W. Ma, and H. Hu, "Real-time queue length estimation for congested signalized intersections," Transportation Research C, vol. 17, no. 4, pp. 412-427, 2009.

[14] X. Wu, H. X. Liu, and D. Gettman, "Identification of oversaturated intersections using high-resolution traffic signal data," Transportation Research C, vol. 18, no. 4, pp. 626-638, 2010.

[15] M. Papageorgiou, J. M. Blosseville, and H. Haj-Salem, "Modelling and real-time control of traffic flow on the southern part of Boulevard Peripherique in Paris: Part II: Coordinated on-ramp metering," Transportation Research A, vol. 24, no. 5, pp. 361-370, 1990.

[16] J. Walmsley, "The practical implementation of SCOOT traffic control systems," Traffic Engineering $\mathcal{E}$ Control, vol. 23, no. 4, pp. 196-199, 1982.

[17] M. J. Norusis, The SPSS Guide to Data Analysis, SPSS, Chicago, Ill, USA, 1990.

[18] J. Pallent, SPSS Survival Manual, SPSS, Chicago, Ill, USA, 2001. 


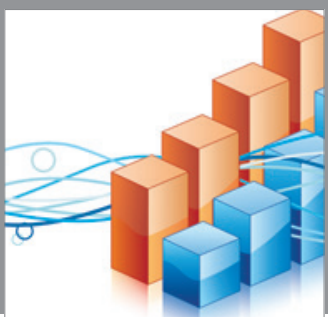

Advances in

Operations Research

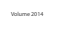

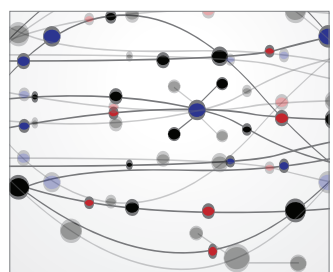

\section{The Scientific} World Journal
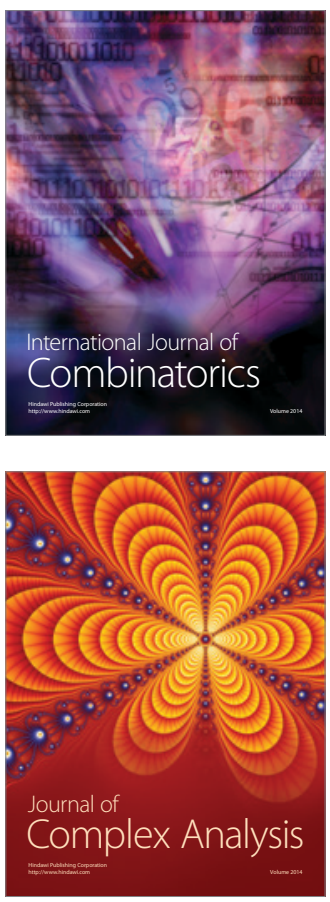

International Journal of

Mathematics and

Mathematical

Sciences
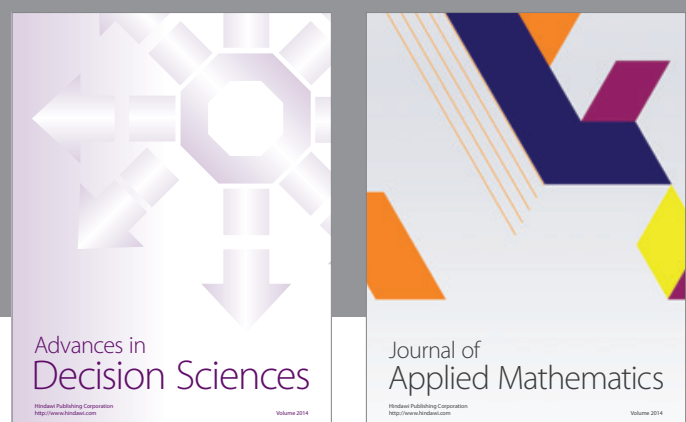

Journal of

Applied Mathematics
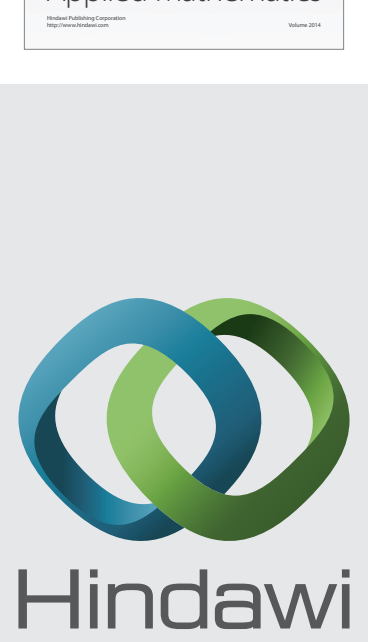

Submit your manuscripts at http://www.hindawi.com
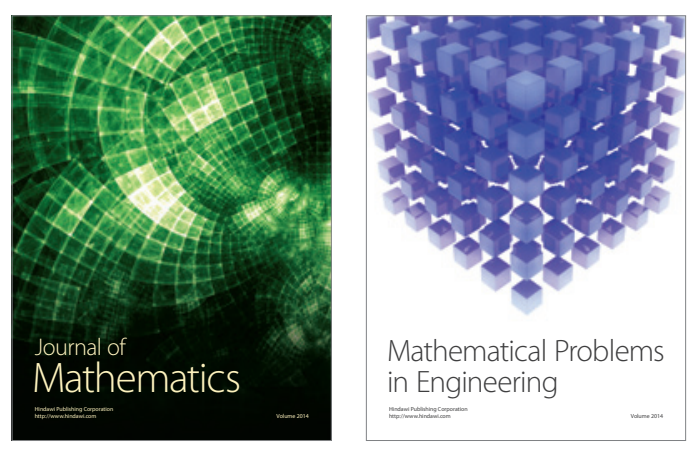

Mathematical Problems in Engineering
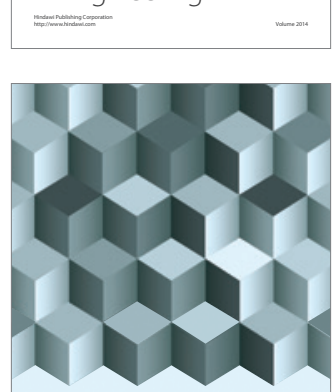

Journal of

Function Spaces
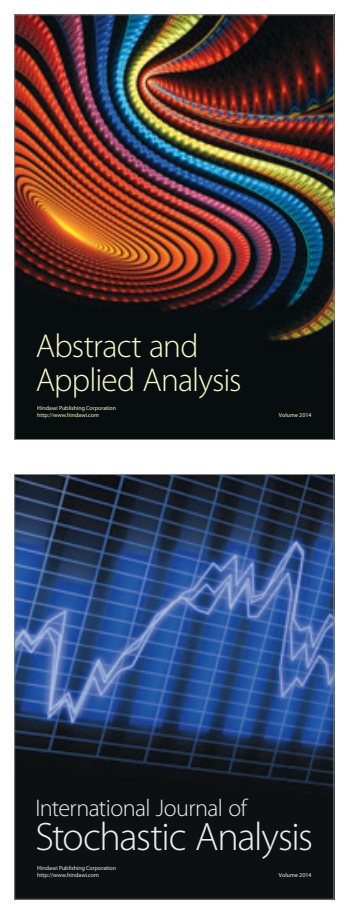

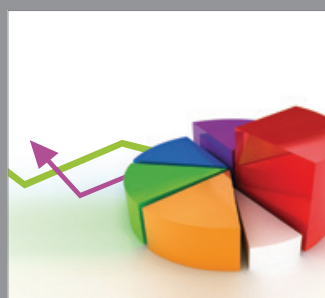

ournal of

Probability and Statistics

Promensencen
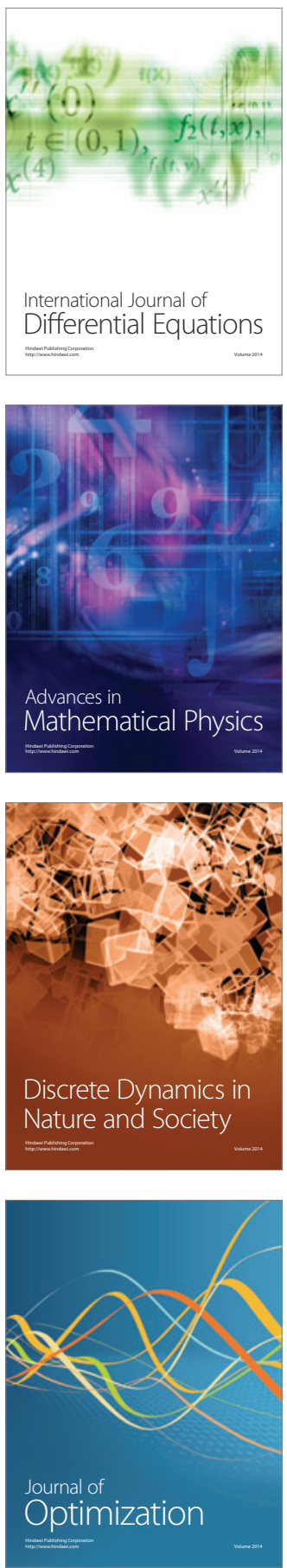